\title{
Selective Target Protein Detection Using a Decorated Nanopore into a Microfluidic Device
}

\author{
Izadora Mayumi Fujinami Tanimoto ${ }^{a, b}$, Benjamin Cressiot ${ }^{c}$, Nathalie Jarroux ${ }^{a}$, Jean Roman ${ }^{b}$, Gilles \\ Patriarche ${ }^{d}$, Bruno Le Pioufle ${ }^{*}$, Juan Pelta ${ }^{* a}$ and Laurent Bacri ${ }^{* a}$ \\ a Université Paris-Saclay, Univ Evry, CNRS, LAMBE, 91025, Evry-Courcouronnes, France \\ b Université Paris-Saclay, ENS Paris-Saclay, CNRS, LuMIn, 91190, Gif-sur-Yvette, France \\ c CY Cergy Paris Université, CNRS, LAMBE, 95000, Cergy, France \\ d Université Paris-Saclay, CNRS, Centre de Nanosciences et de Nanotechnologies, 91120, Palaiseau, France.
}

\section{ARTICLE INFO}

\section{Keywords:}

Solid-state nanopore

Protein sensing

Polymer functionalization

Microfluidics

Nanopore transport

\begin{abstract}
Solid-state nanopores provide a powerful tool to electrically analyze nanoparticles and biomolecules at single-molecule resolution. These biosensors need to have a controlled surface to provide information about the analyte. Specific detection remains limited due to nonspecific interactions between the molecules and the nanopore. Here, a polymer surface modification to passivate the membrane is performed. This functionalization improves nanopore stability and ionic conduction. Moreover, one can control the nanopore diameter and the specific interactions between protein and pore surface. The effect of ionic strength and $\mathrm{pH}$ are probed. Which enables control of the electroosmotic driving force and dynamics. Furthermore, a study of polymer chain structure and permeability in the pore are carried out. The nanopore chip is integrated into a microfluidic device to ease its handling. Finally, a discussion of an ionic conductance model through a permeable crown along the nanopore surface is elucidated. The proof of concept is demonstrated by the capture of free streptavidin by the biotins grafted into the nanopore. In the future, this approach could be used for virus diagnostic, nanoparticle or biomarker sensing.
\end{abstract}

\section{Introduction}

Over the past two decades, nanopore technologies have gathered interest for their potential to address challenges in health (Arima et al., 2018a; Wang et al., 2011), energy (Balme et al., 2017; Bétermier et al., 2020; Huang and Hsu, 2020), and biotechnology (Howorka and Siwy, 2020; Ouldali et al., 2020; Stoloff and Wanunu, 2013). This technology is an electrical sensing technique that detects a single molecule or particle as it passes through the diameter of a nanometer-scale pore. This event corresponds with a specific decrease in ionic current compared to that of the empty pore. Protein channels currently provide the best sensitivity and selectivity (Cressiot et al., 2019; Fahie et al., 2016; Jain et al., 2018; Ramarao and Sanchis, 2013; Wang et al., 2018; Ying and Long, 2019). Moreover, these recombinant nanopores produce highly reproducible data due to their well-defined structure and geometry. Ultra-fast DNA sequencing is one of the most advanced applications of this technology (Branton et al., 2008; Derrington et al., 2010; Jain et al., 2018; Kasianowicz et al., 1996) and recent results pave the way to nanopore protein sequencing (Cressiot et al., 2020; Giamblanco et al., 2020; Ouldali et al., 2020; Restrepo-Pérez et al., 2018). However, certain variables must be considered when using this technology. Protein nanopores are limited to a few nanometers in diameter determined by the specific protein channel used. They are sensitive to the environment, e.g. pressure, organic solvents or chaotropic agents. The lipid bilayer that supports the channel is also fragile. A recent study showed that the membrane fragility could be improved using a chip-based platform capable of sustaining high voltages (Kang et al., 2019; Nardin et al., 2000). With the introduction of material science, new classes of artificial nanopores have been designed (Ma et al., 2020). So-called solid-state nanopores allow for the analysis of DNA conformation (Li et al., 2003; Skinner et al., 2009), protein size (Giamblanco et al., 2018; Wei et al., 2012; Yusko et al., 2017) and conformation (Bacri et al., 2011; Freedman et al., 2013; Waduge et al., 2017), nanoparticles (Bacri et al., 2011) and viruses (Arima et al., 2018b, 2018a). These biomimetic nanopores are stable in different solvents and environments. They are easy to handle and to be produced on a larger scale. Their nanometric diameter can also be tuned to fit the analyte size. There are two types of solid-state nanopores. The first one is the track-etched nanopore drilled in a micrometer thick membrane, leading to the design of specific sensors (Ali et al., 2008; Giamblanco et al., 2020; Lepoitevin et al., 2017, 2016; Lin et al., 2020; Ma et al., 2020, 2019). The second one is drilled in tens of nanometer-thick membranes (Kowalczyk et al., 2011; Li et al., 2001; Roman et al., 2018, 2017; Zhang et al., 2020).

While solid-state nanopores present better mechanical stability than lipid bilayers (Roman et al., 2017), low reproducibility, short lifetime (attributed to high surface energy) (Niedzwiecki et al., 2010; Oukhaled et al., 2011) and, lower

\footnotetext{
* Corresponding author. Bacri).

E-mail addresses: bruno.le-pioufle@ens-paris-saclay.fr (B. Le Pioufle), juan.pelta@univ-evry.fr (J. Pelta), laurent.bacri@univ-evry.fr (L.
} 
a)
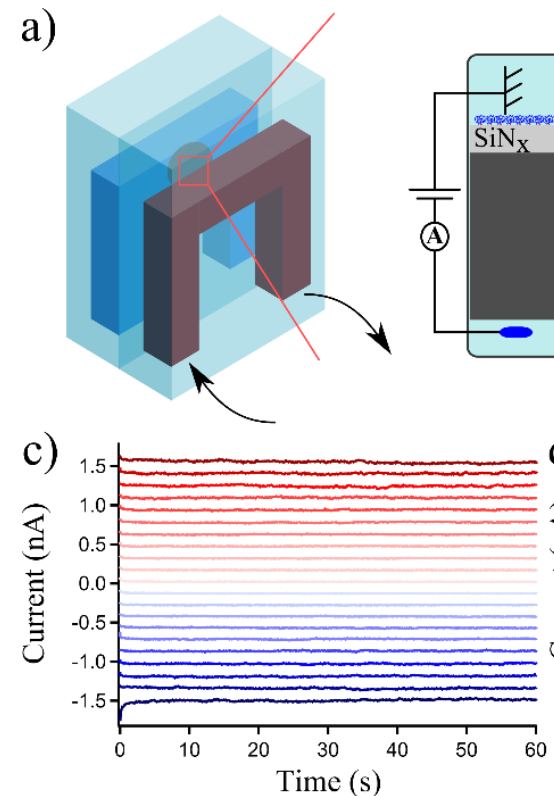

b)

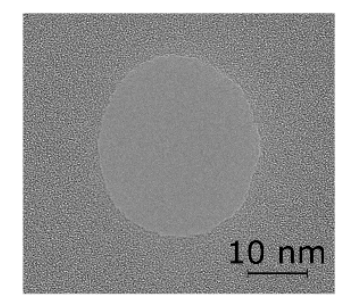

Figure 1. Protein specific capture. a) Schematic of the microfluidic device with a nanopore chip placed on it (left); Sketch of a PEG functionalized nanopore drilled into a $\mathrm{SiN}_{\mathrm{x}}$ based membrane (right). b) High-resolution transmission electron microscopy (HRTEM) picture of a bare 20 nm thick SiN nanopore, $\mathrm{R}_{0}=14 \mathrm{~nm}$. c) A representative sequence of current measurements used to obtain a current vs voltage (I-V) plot; each trace corresponds to a voltage ranging from $-200 \mathrm{mV}$ to $+200 \mathrm{mV}$. d) I-V curve. $\mathrm{SiN}_{\mathrm{x}}$ nanopore, $\mathrm{R}_{0}=14 \mathrm{~nm}, \mathrm{~L}_{0}=20 \mathrm{~nm}$, grafted by $7 \mathrm{kDa} P E G, 0.1 \mathrm{M} \mathrm{NaCl}, 25 \mathrm{mM}$ Tris, $\mathrm{pH}$ 7.4. e) Schematic of the possible forces acting within the nanopore environment under a negative applied voltage. EOF is the electroosmotic flow, and EPF is the electrophoretic force.

sensitivity to detect small species are the major limitations of this technology. There is a need to overcome these limitations. The first strategy is the design of hybrid nanopores to take advantages of both the sensitivity and reproducibility of biological nanopores and, the robustness of solid-state nanopores. In this case, a protein channel is inserted into a thin solid-state membrane (Bentin et al., 2020; Cressiot et al., 2018; Hall et al., 2010) or a polymer membrane (Cabello-Aguilar et al., 2013). The second strategy involves coating the nanopores with a fluid lipid bilayer (Venkatesan et al., 2011; Yusko et al., 2017, 2011). Another one is to control the surface chemistry of the solid-state nanopore by membrane surface functionalization, which allows to increase their lifetime, to control the pore size and to passivate the membrane (Arima et al., 2018b, 2018a; Awasthi et al., 2020; Giamblanco et al., 2018; Lepoitevin et al., 2016; Li et al., 2019; Malekian et al., 2018; Roman et al., 2018, 2017; Wanunu and Meller, 2007). Nevertheless, specific sensing remains a challenge to design new diagnostic tools (Li et al., 2019). Biomimetic nuclear pores have shown the detection of proteins (Kowalczyk et al., 2011; Malekian et al., 2018). Peptide functionalized nanopores are also used to detect influenza virion (Arima et al., 2018a). A similar strategy allowed pulsed amperometric detection of bovine leukemia virus glycoproteins (Ramanaviciene and Ramanavicius, 2004) the immunosensing of bovine leukemia virus (Ramanavicius et al., 2014). These works show the sensitivity of electrical sensing to the functionalization of the electrode surface. In our work, the surface decoration is performed on the nanopore, which becomes a nanoscale probe. Several questions remain open: what is the influence of salt concentration on the polymer size, conformation and mesh size of chains grafted to the pore surface? Is electroosmotic or electrophoretic flow the driving force for the ions and protein dynamics? What is the relation between the polymer layer thickness and ionic permeability? Is it possible to control the protein transport via a specific capture?

Here, we focus on detecting the specific capture and transport of proteins through a nanopore (Fig. 1). The nanopore chip is inserted into a microfluidic device (Fig. 1a, Fig. S1) to facilitate its handling and ease the buffer exchanges.(Roman et al., 2018) The nanopore (Fig. 1b) is functionalized with polymer chains to increase its stability. The polymers are biotinylated to enhance specific single-particle capture and detection of streptavidin (Fig. 1e). The grafting steps are followed and characterized by electrical measurements (Fig. 1c-d), leading to a structural analysis of the functionalized layer. Consequently, we study the transport dynamics through the decorated nanopores. We show how to control this transport according to ionic strength and $\mathrm{pH}$.

\section{Materials and methods}

\subsection{Buffer preparation}

Sodium chloride ( $\mathrm{NaCl}$, Merck, Germany) solutions ranging from $0.1 \mathrm{M}$ to $1 \mathrm{M}$ were prepared at $\mathrm{pH} 7.4$ with $25 \mathrm{mM}$ tris(hydroxymethyl) aminomethane (Tris, Merck, Germany) and pH 6 with $10 \mathrm{mM}$ sodium dihydrogen phosphate monohydrate (NaH2PO4, Avocado, U.K.). The final $\mathrm{pH}$ was adjusted with $\mathrm{HCl}$ and $\mathrm{NaOH}$ solutions using a $\mathrm{pH}$ meter (WTWTM pH meter inoLabTM 720, Xylem Analytics, Germany).

\subsection{Nanopore fabrication}

All silicon nitride membranes were purchased from Nanopore Solutions ${ }^{\circ}$, Portugal. The SiNx chips are $3 \mathrm{~mm}$ large. They are made of a $200 \mu \mathrm{m}$ thick silicon substrate, covered by a $20 \mathrm{~nm}$ thin $\mathrm{Si}_{3} \mathrm{~N}_{4}$ layer. The substrate is etched in its center to obtain a $15 \times 15 \mu \mathrm{m}^{2}$ free-standing $\mathrm{Si}_{3} \mathrm{~N}_{4}$ membrane. The nanopores were drilled with a transmission electron microscope (TEM, Titan Themis) operated at an accelerating voltage of $200 \mathrm{kV}$ according to the standard protocol demonstrated by Storm et al., 2003. In which, by changing the focused electron beam diameter it is possible to control the nanopore size. The beam size can be determined by the full width at half-maximum (FWHM) of the 
Gaussian intensity profile in the transverse focal plane. In this work, the nanopores are ranging from 20 to $35 \mathrm{~nm}$ with a precision lower than $1 \mathrm{~nm}$. Immediately after drilling, the nanopore image obtained by TEM is used to infer its radius $\left(R_{0}\right)$ (Fig. S2).

\subsection{Nanopore functionalization}

Polyethylene glycol (PEG) chains were grafted over the surface of the nanopore chip in a three-step protocol. First, the chip was exposed to a UV/Ozone cleaner (Jelight Company Inc., U.S.A.) for $15 \mathrm{~min}$ per face to generate hydroxyl groups $(-\mathrm{OH})$, activating the surface of the $\mathrm{Si}_{3} \mathrm{~N}_{4}$ membrane. Then, to cover all the active sites of the membrane surface with the (3-chloropropyl) trichlorosilane (CPTS) chains, each face of the chip was incubated for 1 hour at $100 \mathrm{rpm}$ (Rotamax 120, Heidolph Instruments GmbH \& CO, Germany) in a precursor bath of $2.5 \mathrm{mM}$ CPTS (Merck, Germany) in acetone. The final bath, composed of PEG in anhydrous tetrahydrofuran (THF), is applied for 1 hour per face over an orbital shaker. In this step, we use a) only $2.5 \mathrm{mM}$ solution of long $7 \mathrm{kDa}$ PEG (Agilent technologies, U.S.A.) chains, that has been previously described (Roman et al., 2017), or b) a mixture with $1.25 \mathrm{mM}$ of short 500 Da mPEG (Merck, Germany) and $1.25 \mathrm{mM}$ of long 7 kDa PEG chains. The abbreviation $\mathrm{MPEG}$ designates a monomethyl-ether $\left(-\mathrm{OCH}_{3}\right)$ terminated PEG. The treated chips were then rinsed with milli-Q filtered water (Millipore) to remove the non-grafted PEG chains off the membrane. They were stocked in ultra-pure water for further utilization.

The biotin was grafted on the end of the $7 \mathrm{kDa}$ PEG chains (-OH group) through esterification (Gravert and Janda, 1997). The chip was immersed 1 hour per side over an orbital shaker in a dimethyl sulfoxide (DMSO, VWR Chemicals, U.S.A.) solution, containing $3.12 \mathrm{mM}$ biotin (Merck, Germany), $3.12 \mathrm{mM}$ N,N'-Dicyclohexylcarbodiimide (DCC, Merck, Germany) and $0.312 \mathrm{mM}$ 4-pyrrolidinopyridine (Fluka TM analytical, Germany). Then, another rinsing cycle with milli-Q water was performed to remove the non-grafted biotin off the membrane. Functionalized nanopores were stored in ultra-pure water in the refrigerator.

The protein, atto 594-Streptavidin, was purchased from Merck, Germany.

\subsection{Microfluidic device}

The microfluidic device fabrication process has been previously described (Roman et al., 2018). The final device is made of two identical sub-units that form the cis and trans chambers between which the nanopore chip is inserted. Briefly, PolyDiMethylSiloxane (PDMS, Sylgard 184, Dow Corning, U.S.A.) with a 10:1 base to curing agent ratio was used thanks to the conventional soft lithography technology (McDonald and Whitesides, 2002). The mold was 3D-printed (use of an extrusion 3D printer) in PET (polyethylene terephthalate) using a silicon wafer as a substrate. First, the PDMS was poured over the 3D-printed mold to make the channel, and again over a bare wafer to close the channel. Both of them were cured at $75^{\circ} \mathrm{C}$ for $1 \mathrm{~h} 30$. The two parts were sealed together to form one sub-unit by activating the surfaces by $30 \mathrm{sec}$ of low energy air plasma exposure (500-700 mTorr, $15 \mathrm{~W}$ ) from a plasma cleaner (Harrick Plasma, U.S.A). The activated surfaces were then covalently bound after heating at $75^{\circ} \mathrm{C}$ for $20 \mathrm{~min}$. Fluidic access to the channels was made at this step using a biopsy puncher. The two sub-units prepared, as explained above, were then partially bound together. The upper half remains unbound for nanopore chip insertion. Fluidic access tubes were inserted in the pierced holes to act as inlet/outlet of the cis and trans channels. Finally, the apertures are made through the thin layer in between the two sub-units where the chip can be placed to allow the solutions to reach the nanopore.

\subsection{Electric current measurement}

The microfluidic device was filed up with the electrolyte solution. Then, $\mathrm{Ag} / \mathrm{AgCl}$ electrodes were directly inserted in the cis (ground) and trans (working electrode) channels of the microfluidic device to measure the ionic current passing through the nanopore. The current was amplified with an Axopatch 200B (Molecular devices, U.S.A.). A PCle-6251 board (National Instruments, U.S.A.) interfaced with a custom . Net based program was used for data acquisition. Recorded currents were analysed by IGOR Pro (WaveMetrics, U.S.A.). The experiments were carried out in different nanopores $(\mathrm{N}=8)$. The I-V curves were obtained by applying $60 \mathrm{~s}$ long voltage steps $\Delta \mathrm{V}=20$ $\mathrm{mV}$ varying from $-200 \mathrm{mV}$ to $+200 \mathrm{mV}$ at 1 ksamples $/ \mathrm{s}$ and filtered with a Bessel two poles low pass filter at $10 \mathrm{kHz}$. Resistive pulse sensing was performed by applying $120 \mathrm{~s}$ voltage steps at $150 \mathrm{samples} / \mathrm{s}$ and filtered at $10 \mathrm{kHz}$. The final set-up, comprising of the microfluidic device, the nanopore membrane and the amplifier headstage, was placed inside a Faraday cage to avoid electromagnetic interferences. All the measurements were carried out at room temperature and they were repeated 3 times to obtain an averaged value. Thus, for a given concentration of $\mathrm{NaCl}$, we measured the ionic current at least 63 times. Therefore, if we consider the 3 functionalization steps and the 4 different concentrations of $\mathrm{NaCl}$, an average of 756 measurements per nanopore chip was performed.

\section{Results and discussions}

In this study, we focus on developing a device able to detect a specific target molecule, streptavidin, at a singlemolecule scale. To that end, biotin was grafted the free end of each long PEG chain ( $7 \mathrm{kDa}$ PEG), to lead to a biotinstreptavidin complex (Fig. 2), one of the strongest non-covalent interactions known in nature (Weber et al., 1989). The PEG functionalization aims to passivate the membrane, to avoid nonspecific interactions (e.g. experiments with BSA Fig. S3), and to increase the experiment lifetime from a few hours to several months (Fig. S4) (Giamblanco et al., 2018; Roman et al., 2017). Moreover, the nanopore's apparent diameter can be controlled according to the polymer chain size (Roman et al., 2018).

These functionalization steps are followed at the nanometric scale by electrical measurements. After the first 

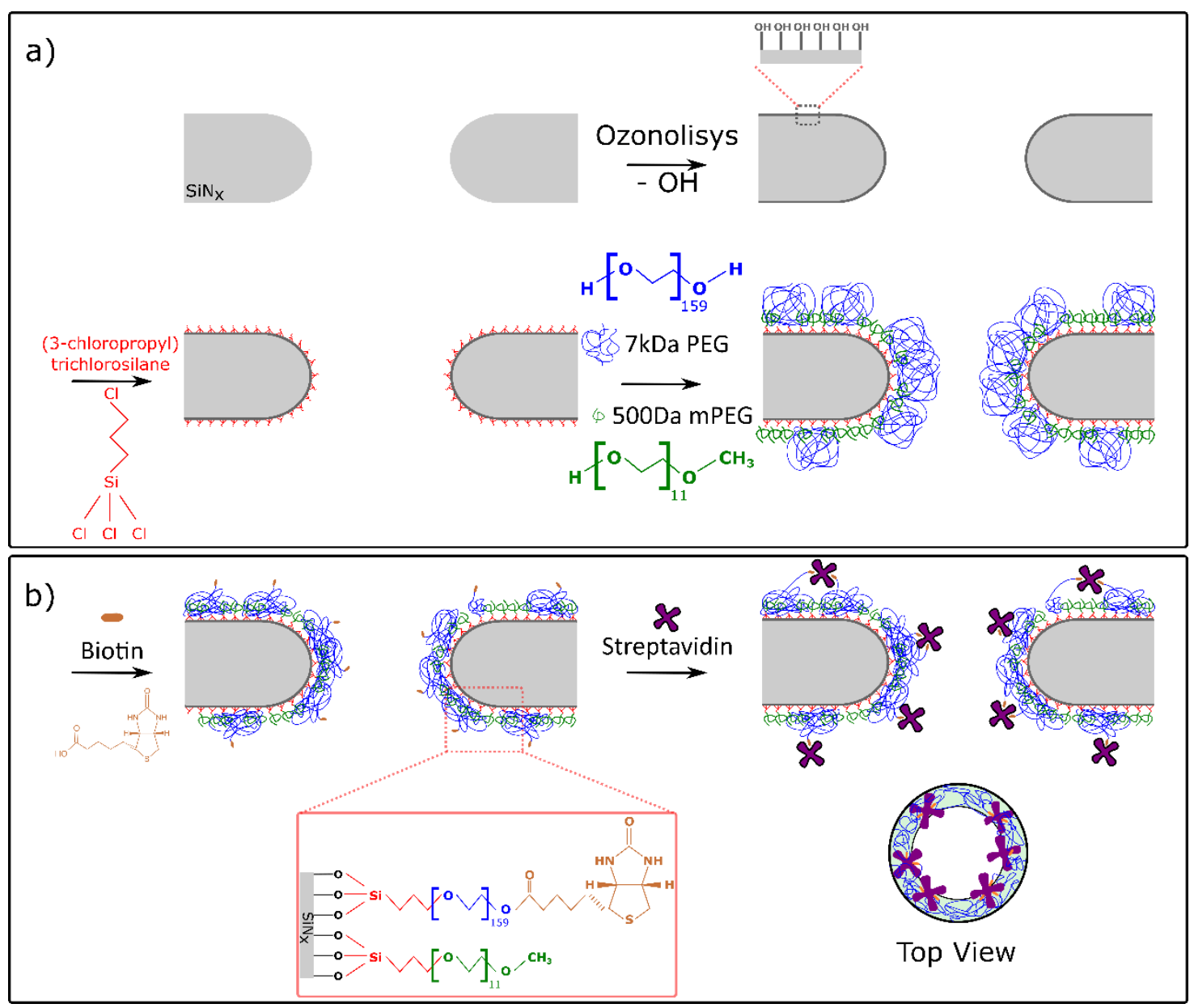

Figure 2. Chemical pathway of the nanopore functionalization. a) Surface passivation and diameter control. The membrane was functionalized with a polymer of 1:1 stoichiometry between 7 kDa PEG and 500 Da mPEG. b) Specific detection mechanism. To specifically capture a target molecule, a bioreceptor, biotin, was grafted at the end of the $7 \mathrm{kDa}$ PEG. On the bottom right a top view of the nanopore system is depicted.

PEG-based grafting, ion current is measured according to voltage to obtain the modified nanopore's current-voltage (I-V) characteristics (Fig. 3a). The linear shape of these curves shows that the driving force does not modify the polymer chain's conformation in response to the applied voltage (Giamblanco et al., 2018; Roman et al., 2018, 2017). The conductance $(G)$ is calculated from the I-V curve slope. $G$ increases with increasing sodium chloride $(\mathrm{NaCl})$ concentration, between 0.1 and $1 \mathrm{M}$.

After nanopore biotinylation as described in Fig. 2, the $I-V$ responses of the nanopore are measured according to ionic strength ( $0.1 \mathrm{M} \leq[\mathrm{NaCl}] \leq 1 \mathrm{M})$ (Fig. 3b). Here, we observe a slight asymmetry in the shape of these I-V curves.

Fig. 3c shows an increase in both the ionic current amplitude and the curve asymmetry between the negative and positive voltage in the presence of streptavidin. We notice that the nanopore conductance also rises with increasing $\mathrm{NaCl}$ concentration. At a constant concentration of $1 \mathrm{M} \mathrm{NaCl}$ (Fig. 3d), we compare the I-V curves measured after PEG functionalization, biotin grafting and streptavidin addition with the values deduced from the calculated conductance of the bare nanopore (see Equation 1). First, we observe a strong decrease in the ionic current from the original bare pore to the PEG grafted pore (Fig. 3d, black vs green). These findings are in good agreement with the presence of a polymer layer covering the inner nanopore (Giamblanco et al., 2018; Kowalczyk et al., 2011; Roman et al., 2017). Then, we observe an increase in conductance after biotin functionalization and streptavidin addition. These results are unexpected and will be discussed below.

Fig. 3 e shows the variation of the nanopore conductance according to the $\mathrm{NaCl}$ concentration. This conductance is positively related to $\mathrm{NaCl}$ concentration (i.e. the conductance increases with rising salt concentration between $0.1 \mathrm{M}$ and $1 \mathrm{M}$ ) as expected in a regime dominated by the ionic transport. This behavior is described by the relation (Balme et al., 2015; Roman et al., 2018):

$G_{\text {ionic }}=\frac{K_{\text {bulk }}}{\frac{L_{0}}{\pi R_{0}^{2}}+\frac{1}{4 R_{0}}}$

where $K_{\text {bulk }}=\left(\mu_{+}+\mu_{-}\right) \cdot e^{2} \cdot c$ is the bulk conductivity, $L_{O}$ is the length of the nanopore, $R_{O}$ is its radius, $e$ is the elementary charge, $c$ is the ion concentration (in ions per $\left.m^{3}\right), \mu_{ \pm}=D_{ \pm} /\left(k_{B} T\right)$ is the bulk ion mobility that is related by the Einstein relation to the ionic diffusion coefficients $(D)$, the Boltzmann constant $k_{B}$ and temperature $T$. Here, the measurements were done using a $\mathrm{NaCl}$ electrolyte with different salt concentrations. Hence, $\mathrm{D}_{\mathrm{Na}^{+}}=$ $1.334 \times 10^{-9} \mathrm{~m}^{2} \mathrm{~s}^{-1}$ and $D_{\mathrm{Cl}^{-}}=2.032 \times 10^{-9} \mathrm{~m}^{2} \mathrm{~s}^{-1}$ at $25^{\circ} \mathrm{C}$. The $1 /\left(4 R_{0}\right)$ term takes into account the access 

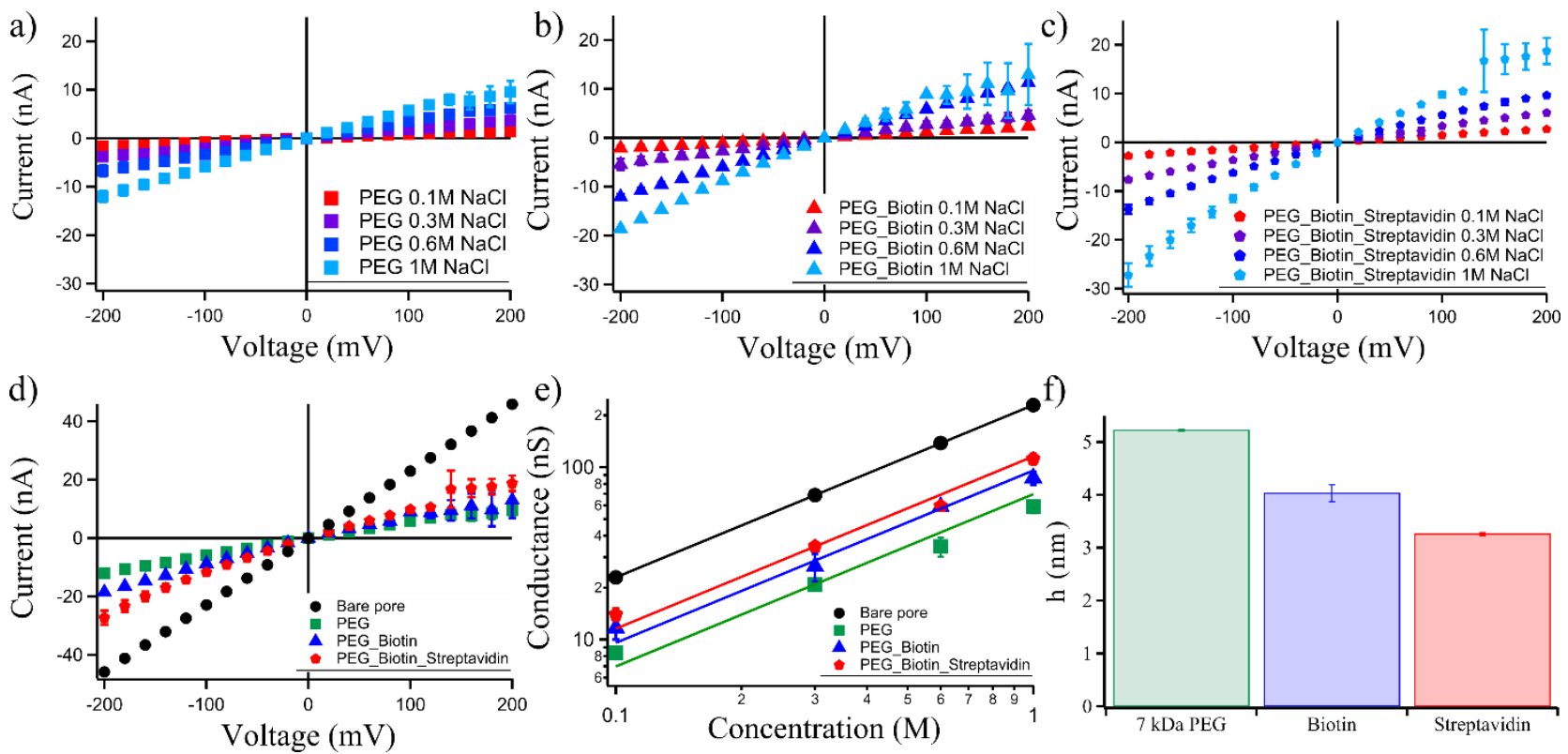

Figure 3. Step by step nanopore functionalization characterization from their ionic conductance according to the ionic strength. a) I-V curves after $7 \mathrm{kDa}$ PEG functionalization according to the ionic strength. b) I-V curves of different $\mathrm{NaCl}$ concentrations after biotin grafting. c) I-V curves after adding the streptavidin into the cis channel. d) I-V curves in $1 \mathrm{M} \mathrm{NaCl}$ in a bare pore (black circles, theoretical calculation), after PEG functionalization (green squares), after biotin grafting (blue triangles) and after streptavidin addition into the cis channel (red pentagons). e) Conductance versus $\mathrm{NaCl}$ concentration after PEG functionalization (green squares), biotin functionalization (blue triangles) and after adding the streptavidin (red pentagons) in the cis channel, at pH 7.4. An increase of the ionic conductance after each functionalization step is observed. Solid lines are calculated from Equation 4. The error bars are computed from three different measurements using the same parameters. f) Grafting thickness after PEG functionalization (green), after biotin grafting (blue) and after streptavidin addition into the cis channel (red). The nanopore size is $\mathrm{R}_{0}=13.25 \mathrm{~nm}$, drilled in a SiN $\mathrm{N}_{\mathrm{x}}$ membrane (thickness $20 \mathrm{~nm}$ ), $0.1 \mathrm{M} \leq[\mathrm{NaCl}] \leq 1 \mathrm{M}, 25 \mathrm{mM}$ Tris, $\mathrm{pH} 7.4$.

resistance near the pore entrance on either side of the membrane (Hall, 1975).

In Fig. 3e, we observe a strong decrease of the conductance due to a decrease in the apparent radius of the nanopore following 7 kDa PEG based functionalization. From which, we compute the functionalized apparent nanopore radius $R_{P E G}^{\prime}=8.03 \pm 0.02 \mathrm{~nm}$, smaller than that of the bare nanopore $R_{0}=13.25 \mathrm{~nm}$ as observed by TEM. The decrease of $5.22 \pm 0.02 \mathrm{~nm}$ is attributed to the PEG layer (Giamblanco et al., 2018; Kowalczyk et al., 2011; Roman et al., 2017). Assuming that the PEG layer thickness $(h)$ is the relevant parameter to characterize the grafted chain conformation whatever the nanopore size and; that the grafting is the same on the membrane and inside the pore, we can calculate the PEG layer thickness. The apparent radius $R^{\prime}$ and length $L^{\prime}$ of the nanopore, after membrane functionalization will become:

$R^{\prime}=R_{0}-h$

$L^{\prime}=L_{0}+2 h$

$R_{o}$ and $L_{o}$ are the initial radius and length of the bare pore, and $h$ is the layer thickness. Thus, from Equation 2 and 3 the Equation 1 can be transformed into:

$G_{\text {ionic }}^{\prime}=\frac{\left(\mu_{+}+\mu_{-}\right) \cdot e^{2} \cdot c}{\frac{L_{0}+2 h}{\pi\left(R_{0}-h\right)^{2}}+\frac{1}{4\left(R_{0}-h\right)}}$

By applying several nanopores $\left(R_{O}=10.2 \mathrm{~nm}, 14 \mathrm{~nm}\right.$, $13.25 \mathrm{~nm}, 13.65 \mathrm{~nm}$ ) grafted by $7 \mathrm{kDa}$ PEG in multiple electrolyte concentrations to the equation above, we obtain an apparent PEG thickness of $h_{7 k D a}=5.1 \pm 0.7 \mathrm{~nm}$ (Fig. 3f).
Nevertheless, the active sites between PEG chains, hydrolyzed in the presence of water, could lead to nonspecific chemical bonds. To rule out this possibility, we used a mixture composed of $7 \mathrm{kDa}$ PEG/500 Da mPEG 1:1 to facilitate the grafting of small 500 Da mPEG chains between each large $7 \mathrm{kDa}$ PEG chain. This grafting, like with $7 \mathrm{kDa}$ PEG chains alone, leads to a decrease of the nanopore conductance (Fig. S5a). Following the same reasoning, we calculate the apparent thickness of the $7 \mathrm{kDa} P E G / 500 \mathrm{Da}$ mPEG grafting layer $h_{\text {mix }}=5.5 \pm 1.5 \mathrm{~nm}$. This result shows that the 500 Da mPEG addition does not influence the 7 kDa PEG chain conformation (Fig. S5b).

After biotinylation of the $7 \mathrm{kDa}$ PEG chains, the conductance linearly increases along with the $\mathrm{NaCl}$ concentration (Fig. 3e). Using Equation 4, the fit leads to an apparent thickness of $h_{\text {Biotin }}=4.03 \pm 0.16 \mathrm{~nm}$ (Fig. 3f) for the pore with biotinylated $7 \mathrm{kDa}$ PEG chains. This value is thinner than the $5.1 \pm 0.7 \mathrm{~nm}$ thickness of the PEG layer before biotinylation.

Considering Equation 4, we fit the curves of Fig. 3e to compute the apparent thickness of the layer composed of the $7 \mathrm{kDa}$ PEG - Biotin - Streptavidin complex, which brings the grafting thickness ( $h_{\text {Streptavidin }}$ ) down to about $3.26 \pm$ $0.03 \mathrm{~nm}$ (Fig. 3f). Similar to what was observed after biotinylation, Fig. 3e-f show, once again, a decrease in thickness of the functionalization layer after streptavidin addition.

These results suggest that the conformation of the PEG chains is modified after biotinylation and streptavidin addition. In ideal solvent conditions, grafted PEG chains have a mushroom-like. The magnitude of the layer thickness is similar to $N^{0.5} a=4.4 \mathrm{~nm}$, where $N$ is the number of monomeric units, and $a$ is the unit length ( $N=159, a=0.35$ $\mathrm{nm}$ for 7 kDa PEG) (de Gennes, 1980). This calculation 

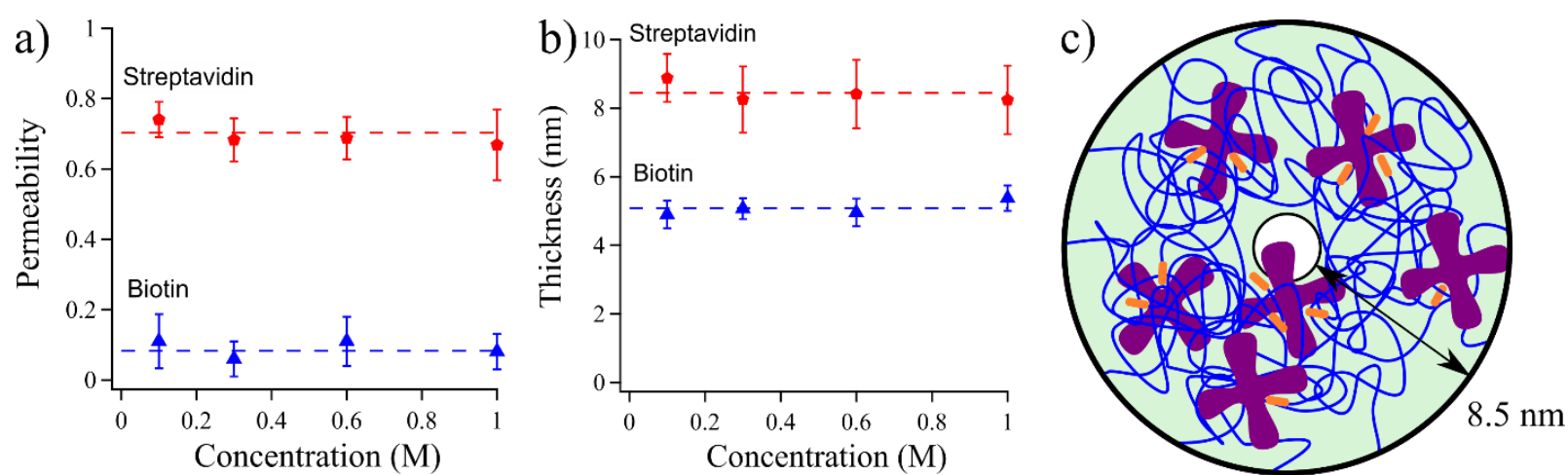

Figure 4. Permeability and thickness of the layers according to ionic strength. a) Permeability of the layer after the biotin grafting $(P=0.09 \pm 2$, red squares), after streptavidin addition ( $P=0.7 \pm 0.03$, blue triangles) b) Thickness of the layer after the biotin grafting ( $h=5.1 \pm 0.2 \mathrm{~nm}$, red squares), after streptavidin addition ( $h=8.5 \pm 0.2 \mathrm{~nm}$, blue triangles). The dotted lines correspond to the average values of each data. $0.1 \mathrm{M} \leq[\mathrm{NaCl} \leq 1 \mathrm{M}$ ], $25 \mathrm{mM}$ Tris, $\mathrm{pH}$ 7.4. c) Structure of a functionalized $20 \mathrm{~nm}$ large nanopore after streptavidin addition.

agrees with the conductance-based nanopore characterization we performed, about $5.1 \pm 0.7 \mathrm{~nm}$ (see above), and with previous works (Giamblanco et al., 2018; Roman et al., 2017). Our observation agrees with the assumption that the polymers could reduce the nanopore diameter. Then, the number of PEG chains grafted on the inner side of the nanopore could be estimated. If we consider a 20 $\mathrm{nm}$ large nanopore, its perimeter is equal to $63 \mathrm{~nm}$. As the PEG size is equal to $5.1 \mathrm{~nm}$, the number of PEG chains along this circumference is estimated to 12 . Given the membrane is also $20 \mathrm{~nm}$ thick, there is only space for 4 layers of PEG leading to 48 PEG chains grafted along the nanopore.

However, the apparent layer thickness surprisingly decreases about $2.11 \pm 1.07 \mathrm{~nm}$, an averaged value for several pores, after biotinylation $\left(h_{P E G}>h_{\text {Biotin }}\right)$. This decrease was an unexpected result because we presumed that the biotin would be grafted within the inner surface of the pore, thus increasing its apparent thickness. A possible interpretation of this result is that the biotin grafted at the end of the $7 \mathrm{kDa}$ PEG chains, could be more attracted to the SiN surface than the solvent, consequently disrupting the conformation of polymer chains. We assumed that the dipole moment of the biotin molecule is attracted to the negative surface of the SiN membrane and could slightly modify the PEG structure. Indeed, Bajgiran et al. (Bajgiran et al., 2020) demonstrated that a biotinylated nanoparticle generates a positive dipole moment and could be used to detect avidin particles. Similarly, a decrease of the apparent thickness was observed when the streptavidin molecules were added ( $\left.h_{P E G}>h_{\text {Biotin }}>h^{\prime}{ }_{\text {Streptavidin }}\right)$. This was possible due to the formation of biotin-streptavidin complex, in which there could be a strong interaction between the negatively charged SiN surface and the apparent positive charge of the biotin at $\mathrm{pH}$ 7.4. These increases in the apparent radius of the nanopore, with the successive functionalization steps, is confirmed in Fig. 3d-e. We found that the conductance increases after each functionalization step whatever the ionic strength between $0.1 \mathrm{M}$ and $1 \mathrm{M}$ $\mathrm{NaCl}$. We verified that even with the increase in ionic current, the apparent diameter did not exceed the diameter of the bare pore (Fig. $3 \mathrm{~d}$-e, black circles), showing that no pore erosion occurred during the experiments.

We suggest the following model to explain these observations. Based on Dekker and colleagues' theory (Ananth et al., 2018), we assume that the structure of the functionalized nanopore is not homogeneous in term of ionic conductivity and is described with three areas: i) the $\mathrm{SiN}$ area considered as an insulator, which conductivity is taken to zero $\left(r=R_{0}\right)$; ii) the functionalized layer with a crown shape $\left(R^{\prime}<r<R_{0}\right)$, characterized by a nonzero conductivity ( $\left.\mathrm{K}_{\text {pore }}\right)$; iii) the central region where there is no polymer chain and which conductivity is equal to the bulk one $\left(K_{\text {bulk }}\right)$ (see above).

The pore conductivity $K_{\text {pore }}$ is obtained by integration of the local ionic conductivity $K(r)$ in the nanopore (Ananth et al., 2018):

$K_{\text {pore }}=\frac{1}{\pi R_{0}^{2}} \int_{r=0}^{R_{0}} 2 \pi r K(r) d r$

where $K(r)=K_{\text {bulk }}$ if $0 \leq r \leq R^{\prime}, K(r)=K_{\text {crown }}$ if $R^{\prime} \leq r<R_{0}$. Then, assuming that the access conductivity $K_{\text {access }}$ is equal to $K_{\text {bulk, }}$, Equation 1 reads: $F$

$$
G=\frac{1}{\frac{L_{0}+2\left(R_{0}-R^{\prime}\right)}{\pi\left[K_{\text {crown }} R_{0}^{2}+\left(K_{\text {bulk }}-K_{\text {crown }} \mathrm{R}^{\prime 2}\right]\right.}+\frac{1}{4 K_{\text {bulk }} R_{0}}}
$$

We could now define the layer permeability as $P=$ $K_{\text {crown }} / K_{\text {bulk }}$ and, considering the layer thickness $h$, the previous relation reads:

$$
G(h, P)=\frac{K_{\text {bulk }}}{\frac{L_{0}+2 h}{\pi\left[P R_{0}^{2}+(1-P)\left(R_{0}-h\right)^{2}\right]}+\frac{1}{4 R_{0}}}
$$

The pore conductance $(G)$ and the pore size $\left(L_{0}, R_{0}\right)$ are considered as constant to graphically solve this relation. In Fig. S6, Equation 7 is plotted by varying both parameters $h$ and $P$ together according to the conductance and the size of three nanopores $\left(R_{0}=10.1 \mathrm{~nm}, R_{0}=10.3 \mathrm{~nm}\right.$ and $R_{0}=$ $13.25 \mathrm{~nm})$. The intersection of these curves leads to the determination of the thickness $(h)$ and the permeability $(P)$ of the functionalized layer after biotin grafting and after streptavidin addition according to the ionic strength (Fig. $4 a-b)$. We could do three observations. Firstly, the permeability and the thickness of the layer do not vary with the ionic strength, between $0.1 \mathrm{M}$ and $1 \mathrm{M}$. Secondly, the low magnitude of the biotinylated layer permeability ( $9 \pm 2 \%$ ) indicate that the biotin grafting slightly modifies the PEG conformation. Nevertheless, the high magnitude of the permeability after the streptavidin addition ( $70 \pm 3 \%$ ) suggests that the streptavidin addition strongly modifies the 

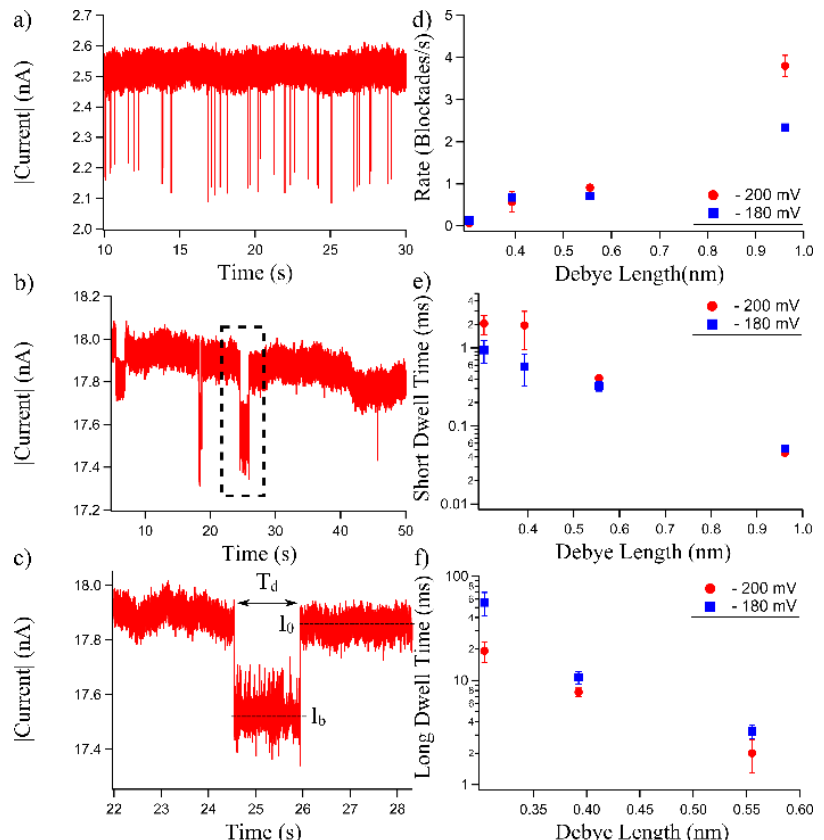

Figure 5. Single-molecule sensing of streptavidin. a-b) lonic current blockades in the presence of $18.8 \mathrm{nM}$ streptavidin in the cis chamber, $\Delta \mathrm{V}=-180 \mathrm{mV}, 0.1 \mathrm{M} \mathrm{NaCl}$ and $1 \mathrm{M} \mathrm{NaCl}$, respectively. c) Focus on one current blockade at $1 \mathrm{M} \mathrm{NaCl}, \Delta \mathrm{V}=-180 \mathrm{mV}, T_{d}$ is the dwell time, and $\Delta \mathrm{I}=\mathrm{I}_{\mathrm{b}}-\mathrm{I}_{0}$ is the current blockade. d) Blockade frequency vs. Debye length $(0.1 \mathrm{M} \leq[\mathrm{NaCl}] \leq 1 \mathrm{M})$. e) Short dwell time vs Debye length. $\mathrm{f})$ Long dwell time vs Debye length. All experiments were performed with a $R_{0}=17.5 \mathrm{~nm}$ nanopore initial radius functionalized with $7 \mathrm{kDa}$ PEG and 500 Da mPEG, and grafted with biotin, $10 \mathrm{mM} \mathrm{NaH}_{2} \mathrm{PO}_{4}$ at $\mathrm{pH} 6$.

PEG structure. Thirdly, the biotin grafting leads to a thickness decrease of about $1 \mathrm{~nm}$, confirming biotinylation does not significantly alter the PEG conformation. However, the streptavidin addition leads to a consistent increase of the thickness until $8.5 \pm 0.2 \mathrm{~nm}$. These results show that the streptavidin molecules interact with the PEG layer leading to a strong change of the chain structure (Fig. 4c). The streptavidin can be bound to four biotins at most. Considering that there are more than 48 biotinylated $7 \mathrm{kDa}$ PEG chains in the $20 \mathrm{~nm}$ large nanopore, we assume that each streptavidin is bound to at least two biotins. Which leads to a polymer network reticulated by the streptavidin. This observation is similar to the one previously performed in track-etched nanopores (Lepoitevin et al., 2016). It shows that the biotinylated nanopore can specifically capture streptavidin (around 48/4 = 12 streptavidin molecules in the $20 \mathrm{~nm}$ large nanopore). The concentration of streptavidin being $18.8 \mathrm{nM}$, we can assume that the majority of the streptavidin is free in solution.

An important issue is whether these functionalized nanopores can perform streptavidin sensing at the singlemolecule level. First, we do not really measure current blockades with the $20.1 \mathrm{~nm}, 20.4 \mathrm{~nm}$ and $20.5 \mathrm{~nm}$ large nanopore (Fig. S7). Considering the model we use, whereas the functionalized layer is $8.5 \mathrm{~nm}$ thick, the central part would be only $3 \mathrm{~nm}$ large. Therefore, streptavidin could not enter this central part because this dimension is smaller than the size of streptavidin and the functionalized layer is too dense to allow streptavidin transport (Fig. 4c). Hence, we doubt that a streptavidin molecule has enough space to enter the nanopore. However, it could be stuck in the nanopore entrance. Subsequently, we use the $26 \mathrm{~nm}$ large nanopore, which is characterized by a $9 \mathrm{~nm}$ large central part, larger than the size of streptavidin. However, the blockade frequency is still relatively low (Fig. S8) when a negative voltage is applied by the electrode in the transside compartment, and the streptavidin is added in the cisside. We did not detect any blockades when we applied a positive bias according to previous works (Yusko et al., 2011; Zhang et al., 2020). Given the isoelectric point (pl) of the streptavidin is 5.5 , this protein is negatively charged at pH 7.5 (Firnkes et al., 2010; Sivasankar et al., 1998). Then, the electrophoretic force (EPF) should not move these proteins from the cis to the trans side. Nevertheless, the SiN based surface is negatively charged at pH 7.5 (Firnkes et al., 2010), leading to an electroosmotic flow (EOF), which can move streptavidin from the cis to the trans side. The long blockade duration we observe could be attributed to the strong electrostatic repulsion between the captured streptavidin in the PEG-biotin layers and the free ones in solution. This repulsion could lead to an increase in the energy barrier of streptavidin confinement through the functionalized nanopore.

To prevent these electrostatic repulsions, i.e. neutralize the protein charge and decrease the streptavidin confinement energy, we decreased the $\mathrm{pH}$ from 7.4 to 6 (Firnkes et al., 2010). Moreover, we decreased the apparent surface charge of the SiN surface (Zeta potential $\zeta$ decreases from $-20 \mathrm{mV}$ at $\mathrm{pH} 7.5$ to $-10 \mathrm{mV}$ at pH 6) (Firnkes et al., 2010) effectively reducing the EOF, which should govern the dynamics through the nanopore (Zhang et al., 2020) (Fig. S9). Now, we study the structure modification of the layer at $\mathrm{pH} 6$. We measure the conductance measurements at $\mathrm{pH} 6$ of $30 \mathrm{~nm}$ and $35 \mathrm{~nm}$ large nanopore: 28.3 $\pm 1.7 \mathrm{nS}$ at $0.3 \mathrm{M} \mathrm{NaCl}, 12.6 \pm 0.96 \mathrm{nS}$ at $0.1 \mathrm{M} \mathrm{NaCl}$, respectively. Following the method described above, we determine the new values of layer thickness $h$ and permeability $P$. The increase of the layer thickness $(h=13.5 \pm 1.7$ $\mathrm{nm})$ and the decrease of its permeability $(P=0.55 \pm 0.06)$ show a modification of its structure due to the $\mathrm{pH}$ decrease (Fig. S10).

We performed this experiment using a $35 \mathrm{~nm}$ diameter nanopore functionalized with biotinylated $7 \mathrm{kDa}$ PEG in the presence of $500 \mathrm{Da} \mathrm{mPEG}$ at $\mathrm{pH} 6$ and $0.1 \mathrm{M} \mathrm{NaCl}$. After streptavidin addition, we observe several blockades at $180 \mathrm{mV}$ applied voltage with a $2.34 \pm 0.06$ blockades/s rate and short duration events ( $<100 \mu \mathrm{s})$ (Fig. 5a). If we increase the electrolyte concentration up to $1 \mathrm{M}$, we observe a decrease of the blockade rate $(<1$ blockades $/ \mathrm{s})$ and the apparition of very long blockades (>>10 ms) (Fig. $5 \mathrm{~b}-\mathrm{c}$ ). We measured the ionic conductance (Fig. S11) at different salt concentrations. We show the ionic current measurements before streptavidin addition in the supporting file (Fig. S12).

We continue with statistical analysis of each blockade, characterized by its duration $T_{d}$, its blockade current $I_{b}$ and the inter-event time between two following blockades $T_{i}$ (Fig. S13). The Fig. $5 \mathrm{~d}$-f shows the results of this analysis according to the Debye length $\left(\kappa^{-1}=\sqrt{\frac{\varepsilon_{0} \varepsilon_{r} k_{B} T}{2 c e^{2}}}\right.$, where $\varepsilon_{0}$ is the vacuum permeability, $\varepsilon_{r}$ the relative permittivity). Which describes the screening length of electrostatic 
interactions. We observe that the blockade rate increases with the Debye length. Two kinds of blockade durations are measured: the short ones (below $2 \mathrm{~ms}$ ) and the long ones (until $90 \mathrm{~ms}$ ). Nevertheless, these blockades are too short to be discriminated at $0.1 \mathrm{M} \mathrm{NaCl}$. The long and short duration times increase as the ionic strength increases.

Consequently, we attempted to understand the physical mechanism involved in the functionalized nanopore after streptavidin addition. From the description of protein transport by Firnkes et al., the effective velocity $V_{\text {eff }}$ of a protein reads:

$V_{e f f}=\frac{\varepsilon_{0} \varepsilon_{r}}{\eta} E\left(\zeta_{\text {protein }}-\zeta_{\text {nanopore }}\right)$

where $E$ the electric field, $\eta$ the solution viscosity. $\zeta_{\text {protein }}$ and $\zeta_{\text {nanopore }}$ are the zeta potential of protein and the nanopore, respectively. At $\mathrm{pH} 6$, the streptavidin could be considered as neutral $\left(\left|\zeta_{\text {protein }}\right|<<\left|\zeta_{\text {nanopore }}\right|\right)$, this equation becomes:

$V_{\text {eff }}=\frac{\varepsilon_{0} \varepsilon_{r}}{\eta} E \zeta_{\text {nanopore }}$

Because the zeta potential of the nanopore is negative and increases with the ionic strength, we assume the absolute value of this zeta potential increases with the Debye length. Then, Equation 9 leads to an increase of the effective velocity magnitude of the streptavidin with $\kappa^{-1}$, which defines the thickness of the counterion flow in the nanopore. In other words, the electroosmotic flow increases with the Debye length. This hypothesis is congruent with the increase of the blockade rate with $\kappa^{-1}$ (Fig. $5 \mathrm{~d}$ ). As the blockade duration could read $T_{d} \simeq \ell / V_{\text {eff }}$ where $\ell=20 \mathrm{~nm}$ is the membrane thickness, the experimental measurements confirm the expected variations of $T_{d}$ (Fig. 5 e $-\mathrm{f}$ ).

Finally, the rebounds of streptavidin failing to enter the nanopore could explain the short blockade durations. The long ones could be explained by the streptavidin going through the functionalized layer, which provides for a partially permeable physical barrier.

\section{Conclusion}

We designed a robust device in which we specifically control the chemistry of solid-state nanopore surfaces, the driving force, the dynamics and the capture of a target protein. It is composed of a nanopore chip inserted in a microfluidic circuit to handle fluids. This device is stable for several days and reusable. It allows ionic current measurements at different salt concentrations and $\mathrm{pH}$ to perform sensing through this nano-channel. This technology is suitable to follow chemical grafting steps at the nanometer scale. The strategy to obtain a selective target protein detection is validated by a hybrid decoration of a nanopore with small polymer chains to avoid nonspecific interactions (Fig. S3 and Fig. S14), and with large ones terminated with biotin groups to increase the specific ones. We consider a model based on the permeability of the functionalized layer in which we monitor the ionic current evolution for all experimental conditions to precisely characterize its structure. This new conformation improves the lifetime of the nanopore (Fig. S15).
The $\mathrm{pH}$ variations allowed us to modify the charge of the protein and of the membrane to control the protein transport through the pore at the single-molecule level. Blockade rates and durations depend on the Debye length, which shows that the electroosmotic flow dominates transport dynamics. The proof of concept was done using the biotin-streptavidin complex. This device is a biomimetic model for specific interactions involved in biology such as antigen-antibody system. It paves the way for the design of a new generation of diagnostics or for applications based on an active decorated nanopore with singleparticle resolution.

\section{Declaration of competing interest}

The authors declare that they have no known competing financial interests of personal relationships that could have appeared to influence the work reported in this paper.

\section{CRediT authorship contribution statement}

Izadora Tanimoto: Investigation, Formal analysis, Data Curation, Visualization, Writing - Original Draft, Writing Review \& Editing. Benjamin Cressiot: Conceptualization, Methodology, Writing - Review \& Editing. Nathalie Jarroux: Methodology, Resources. Jean Roman: Methodology, Software. Gilles Patriarche: Resources. Bruno Le Pioufle: Supervision, Methodology, Resources, Writing - Review \& Editing. Juan Pelta: Supervision, Conceptualization, Validation, Writing - Review \& Editing. Laurent Bacri: Project administration, Funding acquisition, Supervision, Conceptualization, Methodology, Software, Validation, Formal analysis, Data Curation, Visualization, Writing - Original Draft, Writing - Review \& Editing.

\section{Acknowledgements}

We acknowledge financial support from DIM Respore (PhD grants), DEFI Instrumentation aux limites 2016/2017, ANR Epsilomics (17-CE09-0044-02). We thank John T. Gonzalez for careful reading and improving the use of English in the manuscript.

\section{Supplementary data}

Supplementary data to this article can be found online at https://doi.org/

Microfluidic integration; TEM images; Conductance of a nanopore decorated with $7 \mathrm{kDa}$ PEG and $500 \mathrm{Da} \mathrm{mPEG}$ at pH 6 in the presence of $B S A$; Evolution of the ionic conductance of a nanopore decorated with $7 \mathrm{kDa}$ PEG and $500 \mathrm{Da} \mathrm{mPEG}$ at pH 6; lonic conductance after each functionalization step and polymer thickness; Relation between polymer thickness and permeability; Small nanopore decorated with 7 kDa PEG and 500 Da $\mathrm{mPEG}$ at pH 7.4; Large nanopore decorated with $7 \mathrm{kDa}$ PEG at $\mathrm{pH}$ 7.4; Driving forces acting on the nanopore environment; Structure modification of the PEG layer at $\mathrm{pH} \mathrm{6}$; lonic conductance of large nanopore decorated with $7 \mathrm{kDa}$ PEG and $500 \mathrm{Da}$ $\mathrm{mPEG}$ at $\mathrm{pH}$ 6; lonic conductance of large nanopore decorated with $7 \mathrm{kDa}$ PEG and $500 \mathrm{Da} \mathrm{mPEG}$ at pH 6 after biotin functionalization; Nanopore decorated with 7 kDa PEG and 500 Da 
mPEG at pH 6; Conductance of a nanopore decorated with 7 kDa PEG and 500 Da mPEG and biotin-streptavidin complex at $\mathrm{pH} 6$ in the presence of BSA; Evolution of the ionic conductance of a nanopore decorated with $7 \mathrm{kDa}$ PEG and 500 Da mPEG and biotin-streptavidin complex at $\mathrm{pH} 6$.

\section{References}

Ali, M., Yameen, B., Neumann, R., Ensinger, W., Knoll, W., Azzaroni, O., 2008. J. Am. Chem. Soc. 130, 16351-16357.

Ananth, A.N., Mishra, A., Frey, S., Dwarkasing, A., Versloot, R., van der Giessen, E., Görlich, D., Onck, P., Dekker, C., 2018. elife 7, e31510.

Arima, A., Harlisa, I.H., Yoshida, T., Tsutsui, M., Tanaka, M., Yokota, K., Tonomura, W., Yasuda, J., Taniguchi, M., Washio, T., Okochi, M., Kawai, T., 2018a. J. Am. Chem. Soc. 140, 16834-16841.

Arima, A., Tsutsui, M., Harlisa, I.H., Yoshida, T., Tanaka, M., Yokota, K., Tonomura, W., Taniguchi, M., Okochi, M., Washio, T., Kawai, T., 2018b. Sci Rep 8, 16305.

Awasthi, S., Sriboonpeng, P., Ying, C., Houghtaling, J., Shorubalko, I., Marion, S., Davis, S.J., Sola, L., Chiari, M., Radenovic, A., Mayer, M., 2020. Small Methods 4, 2000177.

Bacri, L., Oukhaled, A.G., Schiedt, B., Patriarche, G., Bourhis, E., Gierak, J., Pelta, J., Auvray, L., 2011. J. Phys. Chem. B $115,2890-2898$.

Bajgiran, K.R., Dorman, J.A., Melvin, A.T., 2020. ACS Sens. 5, 2933.

Balme, S., Ma, T., Balanzat, E., Janot, J.-M., 2017. Journal of Membrane Science 544, 18-24.

Balme, S., Picaud, F., Manghi, M., Palmeri, J., Bechelany, M., Cabello-Aguilar, S., Abou-Chaaya, A., Miele, P., Balanzat, E., Janot, J.M., 2015. Sci Rep 5, 10135.

Bentin, J., Balme, S., Picaud, F., 2020. Soft Matter 16, 10021010.

Bétermier, F., Cressiot, B., Di Muccio, G., Jarroux, N., Bacri, L., Morozzo della Rocca, B., Chinappi, M., Pelta, J., Tarascon, J.-M., 2020. Commun Mater 1, 59.

Branton, D., Deamer, D.W., Marziali, A., Bayley, H., Benner, S.A., Butler, T., Di Ventra, M., Garaj, S., Hibbs, A., Huang, X., Jovanovich, S.B., Krstic, P.S., Lindsay, S., Ling, X.S., Mastrangelo, C.H., Meller, A., Oliver, J.S., Pershin, Y.V., Ramsey, J.M., Riehn, R., Soni, G.V., TabardCossa, V., Wanunu, M., Wiggin, M., Schloss, J.A., 2008. Nat Biotechnol 26, 1146-1153.

Cabello-Aguilar, S., Balme, S., Chaaya, A.A., Bechelany, M., Balanzat, E., Janot, J.-M., Pochat-Bohatier, C., Miele, P., Dejardin, P., 2013. Nanoscale 5, 9582.

Cressiot, B., Bacri, L., Pelta, J., 2020. Small Methods 4, 2000090.

Cressiot, B., Greive, S.J., Mojtabavi, M., Antson, A.A., Wanunu, M., 2018. Nat Commun 9, 4652.

Cressiot, B., Ouldali, H., Pastoriza-Gallego, M., Bacri, L., Van der Goot, F.G., Pelta, J., 2019. ACS Sens. 4, 530-548.

Derrington, I.M., Butler, T.Z., Collins, M.D., Manrao, E., Pavlenok, M., Niederweis, M., Gundlach, J.H., 2010. PNAS 107, 16060-16065.

Fahie, M.A., Yang, B., Pham, B., Chen, M., 2016. ACS Sens. 1, 614-622.

Firnkes, M., Pedone, D., Knezevic, J., Döblinger, M., Rant, U., 2010. Nano Lett. 10, 2162-2167.

Freedman, K.J., Haq, S.R., Edel, J.B., Jemth, P., Kim, M.J., 2013. Sci Rep 3, 1638.

de Gennes, P.G., 1980. Macromolecules 13, 1069-1075.

Giamblanco, N., Coglitore, D., Janot, J.-M., Coulon, P.E., Charlot, B., Balme, S., 2018. Sensors and Actuators B: Chemical $260,736-745$.
Giamblanco, N., Janot, J., Gubbiotti, A., Chinappi, M., Balme, S., 2020. Small Methods 4, 1900703.

Gravert, D.J., Janda, K.D., 1997. Chem. Rev. 97, 489-510.

Hall, A.R., Scott, A., Rotem, D., Mehta, K.K., Bayley, H., Dekker, C., 2010. Nat Nanotechnol 5, 874-877.

Hall, J.E., 1975. J Gen Physiol 66, 531-532.

Howorka, S., Siwy, Z.S., 2020. Nature Biotechnology 38, 159160.

Huang, W.-C., Hsu, J.-P., 2020. Electrochimica Acta 353, 136613.

Jain, M., Koren, S., Miga, K.H., Quick, J., Rand, A.C., Sasani, T.A., Tyson, J.R., Beggs, A.D., Dilthey, A.T., Fiddes, I.T., Malla, S., Marriott, H., Nieto, T., O'Grady, J., Olsen, H.E., Pedersen, B.S., Rhie, A., Richardson, H., Quinlan, A.R., Snutch, T.P., Tee, L., Paten, B., Phillippy, A.M., Simpson, J.T., Loman, N.J., Loose, M., 2018. Nature Biotechnology 36, 338-345.

Kang, X., Alibakhshi, M.A., Wanunu, M., 2019. Nano Lett. 19, 9145-9153.

Kasianowicz, J.J., Brandin, E., Branton, D., Deamer, D.W., 1996. PNAS 93, 13770-13773.

Kowalczyk, S.W., Kapinos, L., Blosser, T.R., Magalhães, T., van Nies, P., Lim, R.Y.H., Dekker, C., 2011. Nature Nanotechnology 6, 433-438.

Lepoitevin, M., Bechelany, M., Balanzat, E., Janot, J.-M., Balme, S., 2016. Electrochimica Acta 211, 611-618.

Lepoitevin, M., Ma, T., Bechelany, M., Janot, J.-M., Balme, S., 2017. Advances in Colloid and Interface Science 250, 195-213.

Li, J., Gershow, M., Stein, D., Brandin, E., Golovchenko, J.A., 2003. Nature Mater 2, 611-615.

Li, J., Stein, D., McMullan, C., Branton, D., Aziz, M.J., Golovchenko, J.A., 2001. Nature 412, 166-169.

Li, Q., Ying, Y.-L., Liu, S.-C., Lin, Y., Long, Y.-T., 2019. ACS Sens. 4, 1185-1189.

Lin, C.-Y., Ma, T., Siwy, Z.S., Balme, S., Hsu, J.-P., 2020. J. Phys. Chem. Lett. 11, 60-66.

Ma, T., Balanzat, E., Janot, J.-M., Balme, S., 2019. Biosensors and Bioelectronics 137, 207-212.

Ma, T., Janot, J., Balme, S., 2020. Small Methods 4, 2000366.

Malekian, B., Schoch, R.L., Robson, T., Ferrand -Drake del Castillo, G., Xiong, K., Emilsson, G., Kapinos, L.E., Lim, R.Y.H., Dahlin, A., 2018. Front. Chem. 6, 637.

McDonald, J.C., Whitesides, G.M., 2002. Acc. Chem. Res. 35, 491-499.

Nardin, C., Winterhalter, M., Meier, W., 2000. Langmuir 16, 7708-7712.

Niedzwiecki, D.J., Grazul, J., Movileanu, L., 2010. J. Am. Chem. Soc. 132, 10816-10822.

Oukhaled, A., Cressiot, B., Bacri, L., Pastoriza-Gallego, M., Betton, J.-M., Bourhis, E., Jede, R., Gierak, J., Auvray, L., Pelta, J., 2011. ACS Nano 5, 3628-3638.

Ouldali, H., Sarthak, K., Ensslen, T., Piguet, F., Manivet, P., Pelta, J., Behrends, J.C., Aksimentiev, A., Oukhaled, A., 2020. Nature Biotechnology 38, 176-181.

Ramanaviciene, A., Ramanavicius, A., 2004. Biosensors and Bioelectronics, Special Issue on Synthetic Receptors 20, 1076-1082.

Ramanavicius, A., Oztekin, Y., Ramanaviciene, A., 2014. Sensors and Actuators B: Chemical 197, 237-243.

Ramarao, N., Sanchis, V., 2013. Toxins 5, 1119-1139.

Restrepo-Pérez, L., Joo, C., Dekker, C., 2018. Nat Nanotechnol 13, 786-796.

Roman, J., Français, O., Jarroux, N., Patriarche, G., Pelta, J., Bacri, L., Le Pioufle, B., 2018. ACS Sens. 3, 2129-2137. 
Roman, J., Jarroux, N., Patriarche, G., Français, O., Pelta, J., Le Pioufle, B., Bacri, L., 2017. ACS Appl. Mater. Interfaces 9, 41634-41640.

Sivasankar, S., Subramaniam, S., Leckband, D., 1998. PNAS 95, 12961-12966.

Skinner, G.M., van den Hout, M., Broekmans, O., Dekker, C., Dekker, N.H., 2009. Nano Lett. 9, 2953-2960.

Stoloff, D.H., Wanunu, M., 2013. Curr Opin Biotechnol 24, 699704.

Storm, A.J., Chen, J.H., Ling, X.S., Zandbergen, H.W., Dekker, C., 2003. Nature Materials 2, 537-540.

Venkatesan, B.M., Polans, J., Comer, J., Sridhar, S., Wendell, D., Aksimentiev, A., Bashir, R., 2011. Biomed Microdevices 13, 671-682.

Waduge, P., Hu, R., Bandarkar, P., Yamazaki, H., Cressiot, B., Zhao, Q., Whitford, P.C., Wanunu, M., 2017. ACS Nano 11, 5706-5716.

Wang, Y., Zheng, D., Tan, Q., Wang, M.X., Gu, L.-Q., 2011. Nature Nanotechnology 6, 668-674.

Wang, Y.-Q., Cao, C., Ying, Y.-L., Li, S., Wang, M.-B., Huang, J., Long, Y.-T., 2018. ACS Sens. 3, 779-783.

Wanunu, M., Meller, A., 2007. Nano Lett. 7, 1580-1585.

Weber, P.C., Ohlendorf, D.H., Wendoloski, J.J., Salemme, F.R., 1989. Science 243, 85-88.

Wei, R., Gatterdam, V., Wieneke, R., Tampé, R., Rant, U., 2012. Nat Nanotechnol 7, 257-263.

Ying, Y.-L., Long, Y.-T., 2019. J. Am. Chem. Soc. 141, 1572015729.

Yusko, E.C., Bruhn, B.R., Eggenberger, O.M., Houghtaling, J., Rollings, R.C., Walsh, N.C., Nandivada, S., Pindrus, M., Hall, A.R., Sept, D., Li, J., Kalonia, D.S., Mayer, M., 2017. Nature Nanotechnology 12, 360-367.

Yusko, E.C., Johnson, J.M., Majd, S., Prangkio, P., Rollings, R.C., Li, J., Yang, J., Mayer, M., 2011. Nature Nanotechnology 6, 253-260.

Zhang, Y., Zhao, J., Si, W., Kan, Y., Xu, Z., Sha, J., Chen, Y., 2020. Small Methods 4, 1900893. 